\title{
Performance of a capnodynamic method estimating effective pulmonary blood flow during transient and sustained hypercapnia
}

\author{
Thorir Svavar Sigmundsson ${ }^{1,2}$ - Tomas Öhman ${ }^{1,2}$ - Magnus Hallbäck ${ }^{3}$. \\ Eider Redondo $^{4} \cdot$ Fernando Suarez Sipmann $^{5,6}$ • Mats Wallin ${ }^{2,3}$ - Anders Oldner ${ }^{1,2}$. \\ Caroline Hällsjö Sander ${ }^{1,2} \cdot$ Håkan Björne ${ }^{1,2}$
}

Received: 9 February 2017 / Accepted: 22 April 2017 / Published online: 11 May 2017

(C) The Author(s) 2017. This article is an open access publication

\begin{abstract}
The capnodynamic method is a minimally invasive method continuously calculating effective pulmonary blood flow $\left(\mathrm{CO}_{\mathrm{EPBF}}\right)$, equivalent to cardiac output when intra pulmonary shunt flow is low. The capnodynamic equation joined with a ventilator pattern containing cyclic reoccurring expiratory holds, provides breath to breath hemodynamic monitoring in the anesthetized patient. Its performance however, might be affected by changes in the mixed venous content of carbon dioxide $\left(\mathrm{C}_{\mathrm{v}} \mathrm{CO}_{2}\right)$. The aim of the current study was to evaluate $\mathrm{CO}_{\mathrm{EPBF}}$ during rapid measurable changes in mixed venous carbon dioxide partial pressure $\left(\mathrm{P}_{\mathrm{v}} \mathrm{CO}_{2}\right)$ following ischemia-reperfusion and during sustained hypercapnia in a porcine model. Sixteen pigs were submitted to either ischemia-reperfusion $(n=8)$ after the release of an aortic balloon inflated during $30 \mathrm{~min}$ or to prolonged hypercapnia $(\mathrm{n}=8)$ induced by adding an
\end{abstract}

Electronic supplementary material The online version of this article (doi:10.1007/s10877-017-0021-3) contains supplementary material, which is available to authorized users.

Thorir Svavar Sigmundsson

thorir.sigmundsson@sll.se

1 Department of Perioperative Medicine and Intensive Care, Karolinska University Hospital, Stockholm, Sweden

2 Department of Physiology and Pharmacology, Karolinska Institutet, Stockholm, Sweden

3 Maquet Critical Care AB, Solna, Sweden

4 Department of Intensive Care Medicine, Hospital de Navarra, Pamplona, Spain

5 Hedenstierna's laboratory, Section of Anaesthesiology and Critical Care, Department of Surgical Sciences, Uppsala University, Uppsala, Sweden

6 CIBER de Enfermedades Respiratorias, Instituto de Salud Carlos III, Madrid, Spain instrumental dead space. Reference cardiac output (CO) was measured by an ultrasonic flow probe placed around the pulmonary artery trunk $\left(\mathrm{CO}_{\mathrm{TS}}\right)$. Hemodynamic measurements were obtained at baseline, end of ischemia and during the first $5 \mathrm{~min}$ of reperfusion as well as during prolonged hypercapnia at high and low $\mathrm{CO}$ states. Ischemiareperfusion resulted in large changes in $\mathrm{P}_{\mathrm{v}} \mathrm{CO}_{2}$, hemodynamics and lactate. Bias (limits of agreement) was 0.7 ( -0.4 to 1.8$) \mathrm{L} / \mathrm{min}$ with a mean error of $28 \%$ at baseline. $\mathrm{CO}_{\mathrm{EPBF}}$ was impaired during reperfusion but agreement was restored within $5 \mathrm{~min}$. During prolonged hypercapnia, agreement remained good during changes in $\mathrm{CO}$. The mean polar angle was $-4.19^{\circ}\left(-8.8^{\circ}\right.$ to $\left.0.42^{\circ}\right)$. Capnodynamic $\mathrm{CO}_{\mathrm{EPBF}}$ is affected but recovers rapidly after transient large changes in $\mathrm{P}_{\mathrm{v}} \mathrm{CO}_{2}$ and preserves good agreement and trending ability during states of prolonged hypercapnia at different levels of $\mathrm{CO}$.

Keywords Carbon dioxide - Cardiac output . Intraoperative monitoring $\cdot$ Effective pulmonary blood flow $\cdot$ Capnodynamic $\cdot$ Animal model

\section{Introduction}

Hemodynamic optimisation in high-risk surgery patients using goal directed protocols has shown to improve postoperative outcomes and is assumed to be cost effective [1-5]. A flow based parameter like cardiac output (CO) is ideally used to guide hemodynamic support [3]. Patients accepted for surgery nowadays tend to be older and carry more comorbidity, thus expanding the high-risk group [6]. This underlines the need for a feasible $\mathrm{CO}$ monitor which is both fast and accurate during hemodynamic changes [7]. Several new methods for $\mathrm{CO}$ monitoring have emerged in recent 
years but have different limitations and most have not been adequately evaluated during hemodynamic alterations $[8$, 9].

Alveolar carbon dioxide concentration in expired gas can be easily measured in mechanically ventilated patients. By inducing measurable changes in alveolar carbon dioxide concentration, non-shunted pulmonary blood flow to the lungs can be calculated applying a differential Fick principle [10-12].

Our research group has previously described a capnodynamic method that continuously calculates effective pulmonary blood flow $\left(\mathrm{CO}_{\mathrm{EPBF}}\right)$ based on small changes in $\mathrm{CO}_{2}$ concentration obtained from short expiratory pauses in three out of every nine breaths, conveniently automated by the ventilator [13-15]. $\mathrm{CO}_{\mathrm{EPBF}}$ can be used as a surrogate for $\mathrm{CO}$ when intrapulmonary shunt is low and has shown fair agreement and good trending ability during significant hemodynamic and ventilatory alterations in experimental models [13-16].

Theoretically, the capnodynamic equation is vulnerable to changes in mixed-venous carbon dioxide concentration $\left(\mathrm{CvCO}_{2}\right)$ as the equation system assumes it to remain constant during a measurement cycle. Clinical entities that might cause changes in $\mathrm{CvCO}_{2}$ such as reperfusion and hypercapnia are commonly seen in the perioperative period, for example in aortic and laparoscopic surgery. The aim of this study was to evaluate the performance of the capnodynamic method during ischemia-reperfusion and during prolonged hypercapnia induced by an increased dead space in a porcine model.

\section{Methods}

The study was approved by the Uppsala animal research ethical committee (nr. C 47/15, chairperson Kurt Ek) on April 24th 2015 and performed at the Hedenstierna laboratory in Uppsala University, Sweden.

Hällsjö Sander et al. have previously described the anaesthetic and surgical procedure in detail [13]. Briefly, 16 pigs with a mean weight of $36 \mathrm{~kg}$ (range 32-44 kg) were anaesthetised and mechanically ventilated in a volume-controlled mode with a tidal volume (TV) of $8 \mathrm{~mL} /$ $\mathrm{kg}, \mathrm{FIO}_{2} 0.40$ and PEEP $5 \mathrm{cmH}_{2} \mathrm{O}$ (Servo-i, Maquet Critical Care, Solna, Sweden). Core temperature was maintained at $38-39^{\circ} \mathrm{C}$.

Eight animals underwent the ischemia/reperfusion protocol and eight animals the hypercapnia protocol. The animals used in this study were submitted to other separate experimental steps, not affecting the study protocol, that have been published in abstract form [17].

At the end of the protocol animals were sacrificed by a potassium chloride injection.

\subsection{Instrumentation and equipment}

A $20 \mathrm{G}$ catheter was placed in the carotid artery for arterial pressure monitoring and blood gas sampling. A $7.5 \mathrm{Fr}$ pulmonary artery catheter (Edwards Lifesciences Corp., Irvine, CA, USA) was inserted via the internal jugular vein for analysis of mixed venous blood gases, pulmonary arterial pressure and cardiac output $\left(\mathrm{CO}_{\mathrm{PAC}}\right)$ via thermodilution. A $10 \mathrm{Fr}, 80 \mathrm{~cm}$ thrombectomy catheter (Dispomedica $\mathrm{GmbH}$, Hamburg, Germany) was inserted under ultrasound guidance into the inferior cava vein via the femoral vein to allow controlled preload reduction with balloon inflation.

An ultrasonic flow probe was placed around the pulmonary trunk through a left-sided mini-thoracotomy for $\mathrm{CO}$ measurements $\left(\mathrm{CO}_{\mathrm{TS}}\right)$ ( $\mathrm{T} 401$; Transonic Systems Inc., Ithaca NY, USA).

In the ischemia-reperfusion pigs a $12 \mathrm{Fr}$ stent graft balloon catheter (Reliant ${ }^{\circledR}$, Medtronic Inc. Minneapolis, MN, USA) was inserted into the femoral artery with ultrasound guidance and placed in the abdominal aorta just beneath the diaphragm, verified with fluoroscopy.

Expired carbon dioxide was measured by a mainstream infrared sensor (Capnostat-3, Respironics Inc, Wallingford, CT, USA) and gas flow was analysed by the flow sensor incorporated in the ventilator which was connected to a computer where all the mathematical analysis was carried out with a software written in Matlab ${ }^{\mathrm{TM}}$ (The Mathworks Inc, Natick, MA, USA).

Blood gas analysis was performed by ABL-800FLEX (Radiometer Medical ApS, Brønshøj, Denmark).

Haemodynamic parameters were retrieved into a data acquisition system (Acknowledge, version 3.2.7, Bio Pac Systems, Santa Barbara, CA, USA).

\subsection{Calculations and measurements of cardiac output, shunt and dead space}

The capnodynamic method has previously been reported [15] and a detailed description can be found in the supplementary material. Briefly, a short pause is introduced to the expiratory phase of three out of nine breaths, automatically controlled by the ventilator. The resulting small differences $(4-8 \mathrm{mmHg})$ in the alveolar concentration of $\mathrm{CO}_{2}$ between breaths can be inserted into the capnodynamic equation, describing the mole balance of $\mathrm{CO}_{2}$ transported to and from the lungs. Each breath creates one equation and with a stack of nine equations the $\mathrm{CO}_{\mathrm{EPBF}}$ can be calculated using a least square-error optimization. With each breath the last equation is replaced with the newest allowing a continuous calculation of $\mathrm{CO}_{\mathrm{EPBF}}$, the cardiac output minus the intrapulmonary shunt, with each presented value representing an average of nine preceding breaths. 
The reference method, $\mathrm{CO}_{\mathrm{TS}}$, represents the pulmonary blood flow (shunt included) generated by each cardiac cycle measured at the pulmonary trunk. Each measurement was based on an average of approximately 5-10 s.

$\mathrm{CO}_{\mathrm{PAC}}$, a well-known clinical method to measure $\mathrm{CO}$ (shunt included) was used as a reference in case of technical failure. Values were calculated averaging three intermittent thermodilutions always performed after the $\mathrm{CO}_{\mathrm{TS}}$ and $\mathrm{CO}_{\mathrm{EPBF}}$ measurements to avoid the short acting effect of cold saline on heart rate.

Shunt fraction was calculated using Berggren's formula [18].

Enghoff's dead space was measured using volumetric capnography (NICO monitor, Respironics, Wallingford, CT, USA) [19].

\subsection{Experimental protocol}

After instrumentation and subsequent 15 min stabilisation, precision and baseline $(\mathrm{BL})$ measurements of $\mathrm{CO}_{\mathrm{TS}}$, $\mathrm{CO}_{\mathrm{EPBF}}$ and $\mathrm{CO}_{\mathrm{PAC}}$ were obtained at PEEP $5 \mathrm{cmH}_{2} \mathrm{O}$ and $\mathrm{TV}$ of $8 \mathrm{~mL} / \mathrm{kg}$.

\subsection{Ischemia/reperfusion}

Caudal ischemia was induced in eight pigs by inflation of the aortic balloon for approximately $30 \mathrm{~min}$. The absence of blood flow was confirmed by ultrasound Doppler in the contralateral femoral artery. Severe increases in systemic vascular resistance (SVR) and mean arterial pressure (MAP) during the ischemic period were attenuated with high dose sodium nitroprusside infusion and intermittent beta-blocker boluses. In some animals the aortic balloon was temporarily released and then inflated again if the systolic blood pressure was $>200 \mathrm{mmHg}$, despite maximal pharmacological treatment. Hemodynamic measurements and blood gases were obtained at baseline (BL) and at the end of ischemia, before balloon release. During the reperfusion phase $\mathrm{CO}_{\mathrm{EPBF}}$ and $\mathrm{CO}_{\mathrm{TS}}$ measurements were obtained simultaneously every minute and $\mathrm{CO}_{\mathrm{PAC}}$ at minute 1,3 and 5.

\subsection{Hypercapnia}

Prolonged hypercapnia was induced in eight animals by adding an external dead space at the airway opening between the y-piece and the carbon dioxide sensor, aiming for a 50-60\% increase in $\mathrm{PaCO}_{2}$. Measurements were obtained at baseline, after establishing a stable hypercapnia on average 44 (8) min later and at 7-12 min intervals between baseline, cava balloon inflation, baseline and dobutamine infusion, aiming for $\pm 30 \%$ change in $\mathrm{CO}$. $\mathrm{CO}_{\mathrm{EPBF}}$ and $\mathrm{CO}_{\mathrm{TS}}$ readings were obtained simultaneously.

\subsection{Statistics}

D'Agostino and Pearson omnibus K2 test was used to check data for normal distribution. Results are presented as mean (standard deviation, SD). Proportional bias, i.e., the spread of bias at different $\mathrm{CO}$ levels, was checked with visual assessment and a linear regression. All statistical calculations except for the polar plots and confidence intervals (CI) were performed in Graph Pad Prism (version 6.0 for Windows, Graph Pad Software, La Jolla, CA, USA). For calculation of polar plots an excel sheet for conversion of Cartesian data to polar coordinates was used (kindly provided by Professor L Critchley) and displayed as graphs in Medcalc Statistical Software version 16.8.4 (MedCalc Software bvba, Ostend, Belgium) [20]. Calculations of CI were performed in excel (version 2007) with a t-table representing degrees of freedom, in accordance with the current discussion on comparison of different cardiac output monitors [21].

The animal was allowed to stabilise during and between each hemodynamic measurement during hypercapnia and therefore a correction for repeated measurements was not applied [21, 22].

\subsection{Inherent precision}

Inherent precision (defined as twice the coefficient of variation $\left(\mathrm{CV}=\mathrm{SD}_{\text {method }} /\right.$ mean $\left.\mathrm{CO}_{\text {method }}\right)$ of $\mathrm{CO}_{\mathrm{EPBF}}$ was calculated from 10 measurements obtained at 1 min intervals in each animal at baseline conditions [23]. The previously reported precision for $\mathrm{CO}_{\mathrm{TS}}$ was $\pm 10 \%$ [24] and between \pm 8 and $\pm 24 \%$ for $\mathrm{CO}_{\mathrm{PAC}}$ depending on different haemodynamic conditions, ventilation, temperature and positioning of the catheter per se $[25,26]$. Precision for $\mathrm{CO}_{\mathrm{EPBF}}$ was $\pm 14 \%$ during steady state conditions in our previous study [15].

\subsection{Absolute values}

Bland Altman methodology was used to measure the mean difference (bias) and the precision (levels of agreement) between $\mathrm{CO}_{\mathrm{EPBF}}$ and $\mathrm{CO}_{\mathrm{TS}}$ [27-29]. Proportional bias was checked with regression analysis to see if the slope deviated from zero. Due to small sample size the mean error (ME), also known as percentage error, used to estimate the precision of agreement was calculated as $100 \% \times \mathrm{t}_{\alpha, \mathrm{n}-1} \times \mathrm{SD}_{\text {bias }}$ I mean $\mathrm{CO}_{\text {ref }}$, where $\mathrm{t}_{\alpha, n-1}$ is the $\mathrm{t}$-value corresponding to the degrees of freedom $(n-1)$ and a type I error $(\alpha)$ of 0.05 $[21,29]$.

Confidence intervals (CI) were calculated for all analyses, as described above. A priori, $\mathrm{CO}_{\mathrm{EPBF}}$ was considered interchangeable to $\mathrm{CO}_{\mathrm{TS}}$ if mean error was less than $30 \%$ [29], although less than $45 \%$ might be considered in 
settings of extensive hemodynamic changes or apparent advantage of the reference method [30].

\subsection{Trending ability}

The four-quadrant and the polar plot methodology were used to assess the agreement between test and reference methods regarding the direction and magnitude of the change [20]. Concordance rate for the four-quadrant plot was calculated as the number of data points in the two quadrants of agreement divided by the total number of data points, expressing the agreement between the paired delta$\mathrm{CO}$ values for both methods [20]. Concordance rate for the polar plot was calculated as the number of data points within the radial limits of agreement of $\pm 30^{\circ}$ divided by the total number of data points [20].

Because of the high precision of the reference method, $<20 \%$, an exclusion zone of $10 \%$ was used [31]. We considered a concordance rate of $>92$ and $>90 \%$ calculated by the four-quadrant plot and the polar plot methodology as good, respectively [32]. An angular bias smaller than $\pm 5^{\circ}$ indicated sufficient calibration between the test and the reference method [20, 32].

\section{Results}

All animals survived the experimental protocol that resulted in notable hemodynamic changes. The calculated inherent precision of $\mathrm{CO}_{\mathrm{EPBF}}, \mathrm{CO}_{\mathrm{TS}}$ and $\mathrm{CO}_{\mathrm{PAC}}$ during initial baseline conditions was 8, 4 and $10 \%$, respectively. All data were normally distributed. Proportional bias between $\mathrm{CO}_{\mathrm{EPBF}}$ and $\mathrm{CO}_{\mathrm{TS}}$ was undetected in all interventions except during preload reduction in the hypercapnia protocol.

\subsection{Ischemia/reperfusion}

At the end of ischemia, a decrease in $\mathrm{PvCO}_{2}$ and increase in serum lactate were observed. Immediately after balloon release, $\mathrm{PaCO}_{2}$ and $\mathrm{PvCO}_{2}$ increased dramatically and remained high during the initial reperfusion phase (Table 1). Cardiac output varied throughout the protocol (Table 1) and other hemodynamic parameters were largely affected (Table 2). $\mathrm{CO}_{\mathrm{EPBF}}$ and $\mathrm{CO}_{\mathrm{TS}}$ showed bias (LoA) 0.7 ( -0.4 to 1.8$) \mathrm{L} / \mathrm{min}$ and ME $28 \%$ at baseline. The performance of $\mathrm{CO}_{\mathrm{EPBF}}$ deteriorated immediately after balloon release but was restored gradually over the next $5 \mathrm{~min}$ (Fig. 1; Table 2).

\subsection{Hypercapnia}

During prolonged hypercapnia, $\mathrm{PaCO}_{2}$ and $\mathrm{PvCO}_{2}$ increased from $5.6(0.42)$ to $9.2(0.5) \mathrm{kPa}$, and $7.41(0.69)$ to $10.5(0.91) \mathrm{kPa}$, respectively. See Table 2 for all hemodynamic, ventilatory and metabolic values.

At hypercapnia baseline conditions, $\mathrm{CO}_{\mathrm{EPBF}}$ bias (LoA) was $0.5(-0.5$ to 1.4$) \mathrm{L} / \mathrm{min}$ and $\mathrm{ME}$ was $21 \%$.

The corresponding values at preload reduction and inotropic stimulation were $0.4(-0.7$ to 1.4$)$ and $0.04(-2.8$ to 2.8) $\mathrm{L} / \mathrm{min}$, respectively. ME was $38 \%$ in both conditions. See Fig. 2 for visual assessment of the Bland Alman plot during hypercapnia and Table 1 for Bland Altman values for all interventions separately.

\subsection{Trending during hypercapnia}

The concordance rate for $\mathrm{CO}_{\mathrm{EPBF}}$ and $\mathrm{CO}_{\mathrm{TS}}$ following preload reduction and inotropic stimulation was $100 \%$ according to both four quadrant and polar plot methodologies. The mean $(95 \% \mathrm{CI})$ polar angle was $-4.19^{\circ}\left(-8.8^{\circ}\right.$ to $0.42^{\circ}$ ) (Fig. 3).

Table 1 Cardiac output (L/min) and Bland-Altman values for $\mathrm{CO}_{\mathrm{TS}}$ and $\mathrm{CO}_{\mathrm{EPBF}}$ at baseline and during different interventions with confidence intervals (CI) for bias and upper/lower level of agreement (LoA) and mean error (ME)

\begin{tabular}{|c|c|c|c|c|c|c|c|c|}
\hline Condition (n) & $\mathrm{CO}_{\mathrm{EPBF}}(\mathrm{L} / \mathrm{min})$ & $\mathrm{CO}_{\mathrm{TS}}(\mathrm{L} / \mathrm{min})$ & Bias & CI & LoA & CI lower (LoA) & CI upper (LoA) & $\operatorname{ME}(\%)$ \\
\hline Baseline (8) & $4.5(0.4)$ & $3.9(0.5)$ & 0.7 & 0.3 to 1.1 & -0.4 to 1.8 & -1.1 to 0.3 & 1.1 to 2.5 & 28 \\
\hline End of iscemia (6) & $3.9(0.5)$ & $5.4(0.6)$ & -1.5 & -2.3 to -0.7 & -3.6 to 0.5 & -4.9 to -2.2 & -0.8 to 1.9 & 36 \\
\hline Reperfusion 1 min (8) & $18.8(3.4)$ & $4.2(1.0)$ & 14.6 & 11.8 to 17.4 & 6.5 to 22.7 & 1.7 to 11.3 & 17.8 to 27.5 & 186 \\
\hline Reperfusion $3 \min (8)$ & $6.0(1.5)$ & $4.9(1.0)$ & 1.1 & -0.4 to 2.6 & -3.3 to 5.5 & -5.9 to -0.7 & 2.9 to 8.1 & 87 \\
\hline Reperfusion $5 \min (8)$ & $5.1(0.8)$ & $5.1(0.8)$ & 0.03 & -0.7 to 0.8 & -2.2 to 2.2 & -3.5 to -0.9 & 0.9 to 3.5 & 42 \\
\hline Baseline (8) & $3.6(0.5)$ & $3.1(0.6)$ & 0.6 & 0.1 to 1.0 & 0.8 to 1.9 & -1.6 to 0.0 & 1.1 to 2.7 & 42 \\
\hline Hypercapnia (24) & $5.0(0.7)$ & $4.5(0.5)$ & 0.5 & 0.3 to 0.7 & -0.5 to 1.4 & -0.8 to -0.1 & 1.1 to 1.7 & 21 \\
\hline Caval occlusion (8) & $3.1(0.5)$ & $2.7(0.2)$ & 0.4 & 0.0 to 0.7 & -0.7 to 1.4 & -1.3 to -0.1 & 0.8 to 2.1 & 38 \\
\hline Dobutamine (8) & $7.0(1.0)$ & $7.04(0.9)$ & 0.04 & -1.0 to 0.9 & -2.8 to 2.7 & -4.4 to -1.1 & 1.1 to 4.3 & 38 \\
\hline
\end{tabular}




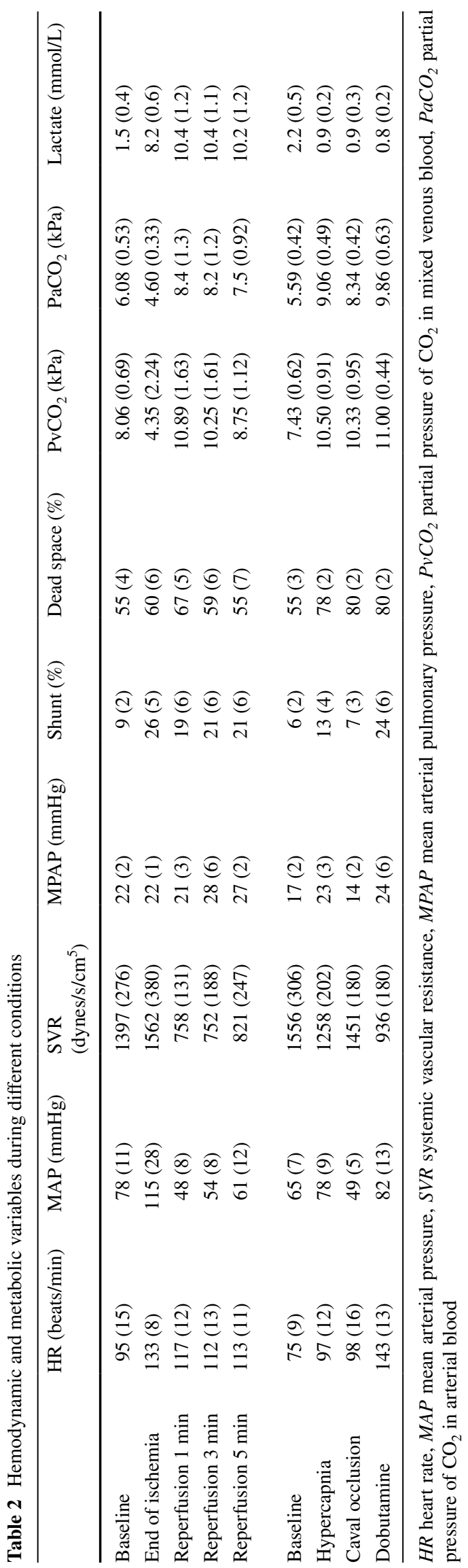

\section{Discussion}

We have evaluated the performance of a capnodynamic method to estimate $\mathrm{CO}_{\mathrm{EPBF}}$ in a porcine model of ischemia/ reperfusion and hypercapnia. As expected, agreement was impaired during reperfusion due to the sudden flux of carbon dioxide into the circulation. However, agreement was restored within $5 \mathrm{~min}$. Prolonged hypercapnia did not impair agreement or trending ability of this method.

Anaesthetists working in the operating room are well aware that rapid physiological changes are to be expected. However, the performance of most non-invasive CO monitors has been found to be less reliable during rapid changes in vascular volume and resistance, a common feature during major surgery $[8,9]$.

The Fick's principle, using elimination of $\mathrm{CO}_{2}$ from the lungs and the difference in concentration of $\mathrm{CO}_{2}$ in mixed venous and arterial blood is a well proven gold standard to calculate $\mathrm{CO}$, although unsuitable for clinical use [33]. Modifications of Fick's principle based on end tidal $\mathrm{CO}_{2}$ measurements, assuming a steady state in $\mathrm{C}_{\mathrm{V}} \mathrm{CO}_{2}$, have been used to calculate effective pulmonary blood flow indirectly, which equals $\mathrm{CO}$ minus the shunt flow [34]. These indirect methods are dependent on induced alterations in alveolar $\mathrm{CO}_{2}$ accomplished by three means; (1) changes in the alveolar ventilation as originally described by Gideon et al. [10] and applied with modifications in our capnodynamic method, (2) changes in dead space as originally described by Capek et al. [35] and used in the NICO system with different software versions $[36,37]$ and (3) adding carbon dioxide to the inspiratory gas.

The capnodynamic method is continuous with short response time and needs no additional devices mounted to the y-piece, as opposed to the NICO monitor. Originally, inspiratory pauses where used to obtain the desired changes in alveolar ventilation. Good trending was observed during hemodynamic changes and lung injury but agreement (bias) and precision (LoA) was affected at higher PEEP and shunt levels in a porcine model [13, 14]. Following refinement changing to expiratory instead of inspiratory pauses, both agreement and precision improved when compared with the highly invasive ultrasonic flow probe and thermodilution based methods during significant hemodynamic and ventilatory changes [13-16].

A prerequisite for the capnodynamic equation is a stable concentration of $\mathrm{C}_{\mathrm{v}} \mathrm{CO}_{2}$ during the measurement cycle. Therefore, we wanted to test the hypothesis that large alterations in venous concentration of carbon dioxide, as might be seen during major surgery, laparoscopy and lung protective ventilation would impair the performance of $\mathrm{CO}_{\mathrm{EPBF}}$. Rapid influx of carbon dioxide into the circulation is to be expected during vascular surgery following periods of ischemia, whereas in laparoscopic and robotic assisted 


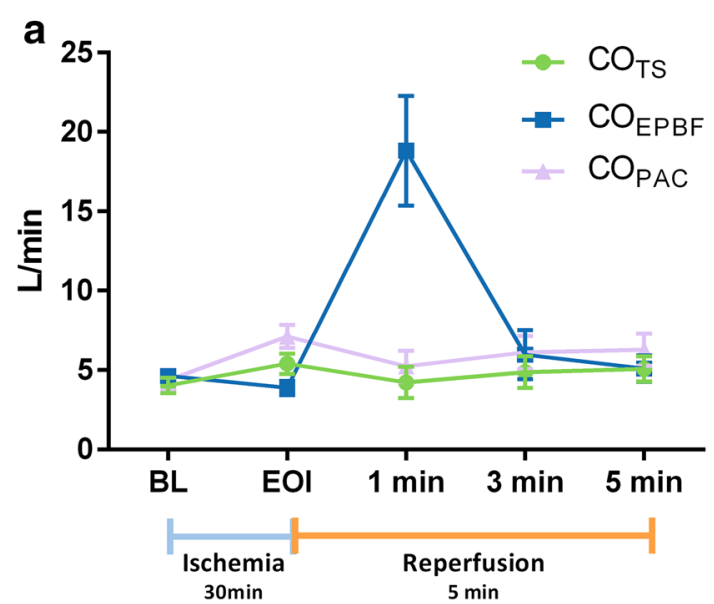

Fig. 1 Timeline showing a mean (SD) values for $\mathrm{CO}_{\mathrm{EPBF}}, \mathrm{CO}_{\mathrm{TS}}$ and $\mathrm{CO}_{\mathrm{PAC}}$ from baseline (BL) to end of ischemia (EOI) approximately 30 min later and at minute 1,3 and 5 after reperfusion, and $\mathbf{b}$ continu-

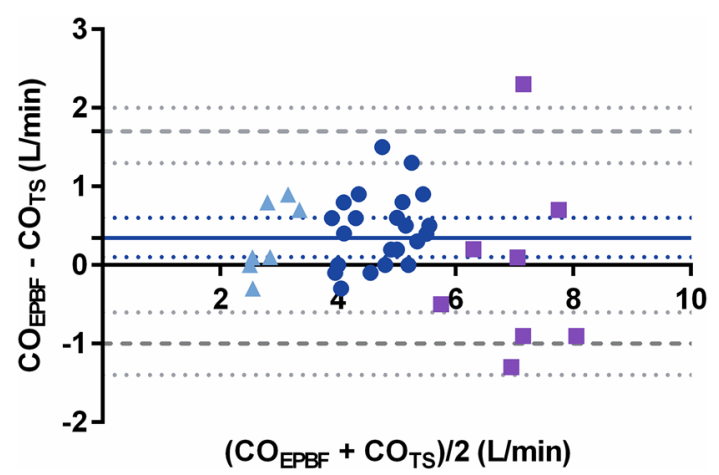

Fig. 2 Bland-Altman plot showing 40 paired values for $\mathrm{CO}_{\mathrm{EPBF}}$ vs $\mathrm{CO}_{\mathrm{TS}}$ during dead space induced hypercapnia at baseline (blue dots), low CO (light blue triangles) and high CO (purple quadrants). Bias is represented with a whole blue line with corresponding CI (blue dotted lines) and levels of agreement (LoA) are shown with broken grey lines with corresponding CI (grey dotted lines)

surgery, insufflation of carbon dioxide induces prolonged hypercapnia. These clinical situations are common features in the operating theatre. The current protocol was designed to evaluate the performance of $\mathrm{CO}_{\mathrm{EPBF}}$ in an animal model mimicking these clinical scenarios.

In the current study, ischemia and subsequent reperfusion were accomplished by aortic occlusion simulating suprarenal clamping during aortic surgery. The balloon inflation created severe hemodynamic changes in the animal, including a sudden massive increase in systemic vascular resistance demanding treatment with vasodilatory and rate regulatory drugs. As a result, pulmonary shunt increased from 9 to $26 \%$ at the end of ischemia and although decreasing during reperfusion, shunt remained elevated from baseline. Since $\mathrm{CO}_{\mathrm{EPBF}}$ does not detect b Trace from one animal

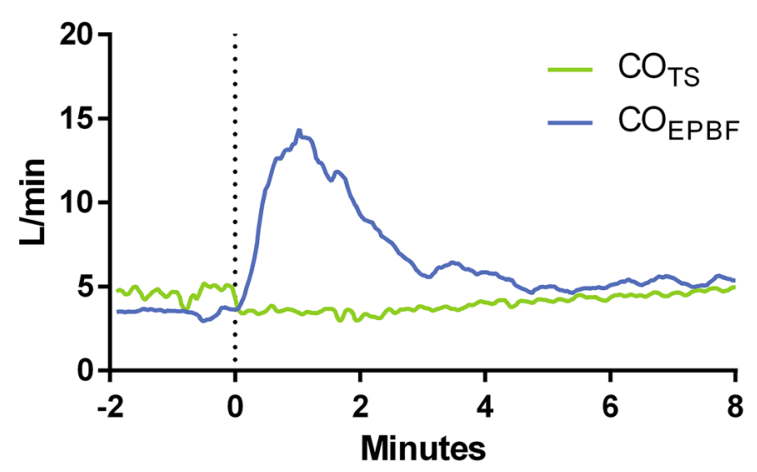

ous values from one animal for $\mathrm{CO}_{\mathrm{EPBF}}$ and $\mathrm{CO}_{\mathrm{TS}}$ (not possible with $\mathrm{CO}_{\mathrm{PAC}}$ ) from 2 min before balloon release (vertical broken line) and up to 8 min after reperfusion

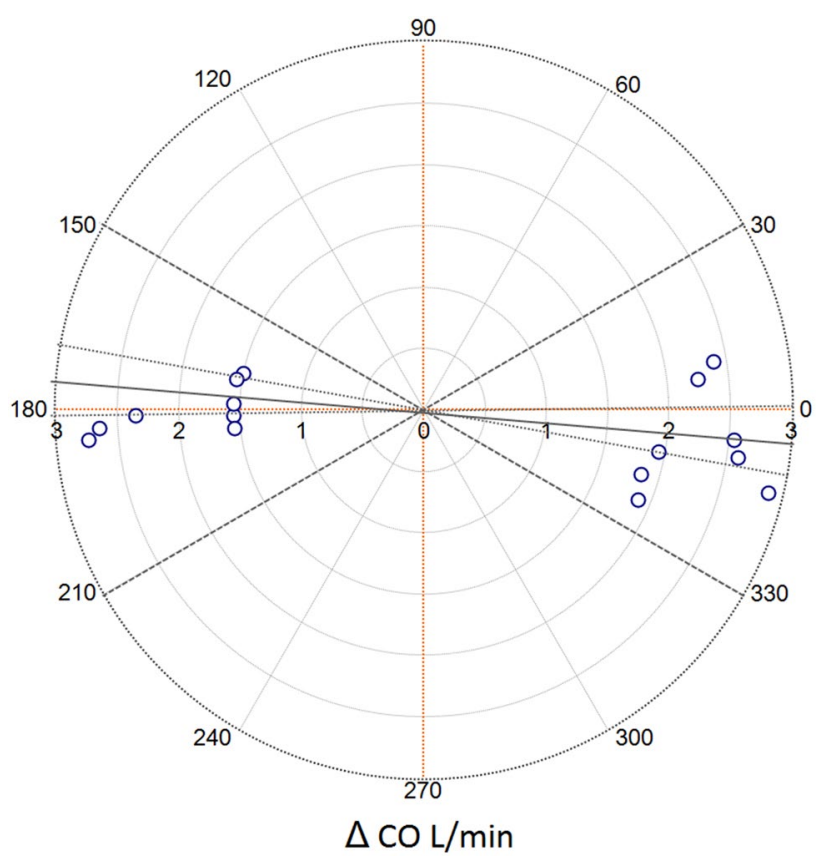

Fig. 3 Polar plot for $\mathrm{CO}_{\mathrm{EPBF}}$ with $\mathrm{CO}_{\mathrm{TS}}$ as the reference method during dead space induced hypercapnia. The radial length is the mean of the pairwise delta values of the reference method and the test method (L/min) and is shown with a whole black line with corresponding CI (dotted lines). Data spread closely to the polar axis (orange dotted lines) indicate good trending

shunt flow, this contributed to the underestimation of $\mathrm{CO}$ at the end of ischemia (see Fig. 1; Table 1). As the capnodynamic equation requires a stable concentration of venous carbon dioxide during the measurement cycle in order to be accurate, $\mathrm{CO}_{\mathrm{EPBF}}$ increased erroneously following the aortic balloon release due to the resulting 
dramatic rise in alveolar concentration of carbon dioxide. Despite the massive reperfusion, the performance of $\mathrm{CO}_{\text {EPBF }}$ was nearly fully restored within $5 \mathrm{~min}$.

Prolonged hypercapnia was induced by adding dead space to the breathing circuit to maintain similar tidal volumes and respiratory rate and to mimic the hypercapnia often seen in laparoscopic surgery. Cardiac output, dead space, shunt, $\mathrm{PaCO}_{2}$ and $\mathrm{PvCO}_{2}$ increased as expected but did not affect bias, LoA or ME (see Table 2). These results indicate that the concentration of carbon dioxide per se does not impair the performance of the method in contrast to sudden changes. The performance of many $\mathrm{CO}$ monitoring techniques is impaired during rapid hemodynamic changes and requires frequent calibration in order to maintain accuracy. The capnodynamic method recuperated within 5 min after reperfusion. This time span is comparable to the duration of the calibration procedures of the thermodilution based CO monitors [30, 38].

The capnodynamic method also includes an internal control function that detects major incongruities that do not fit with the ideal capnodynamic equation. The inherent control function, not used in this study, has the potential to filter out unstable values as those seen in the reperfusion phase, supporting the clinician with only stable calculations of pulmonary blood flow.

In the statistical analysis we adapted to the most recent guidelines on comparing different cardiac output monitors [21]. Taken our previous studies and animalresearch ethics into account we used as few animals as possible, despite an expected effect on confidence intervals and higher risk for proportional bias. The physiological stresses imposed on the animals in this study were extreme and the results needs to be interpreted in this context. At baseline the capnodynamic method showed acceptable bias, LoA and ME. Five minutes after massive reperfusion, notable increase in $\mathrm{CO}$ and large reduction in SVR the bias was almost zero but LoA and ME had increased. We believe this is acceptable for a continuous minimally invasive method complimentary to mechanical ventilation in anesthetized subjects, as long as trending is preserved, as previously suggested by Peyton and Chong [30].

This study has several limitations. As the capnodynamic method measures carbon dioxide in expired gas, the mixedvenous carbon dioxide bypassing the lungs i.e., shunted, is not detected. In the current study, we have utilized $\mathrm{CO}$ as a reference for comparison. Since the reference method and the test method do not measure identical physiological variables, a difference is to be expected. Our previous studies have shown that $\mathrm{CO}_{\mathrm{EPBF}}$ has acceptable agreement when shunt is below $20 \%$. At higher shunt fractions the agreement is impaired, however, the trending ability is still reliable [13-15].
The major hemodynamic changes in the protocol required intermittent intense pharmacologic support. This resulted in inter-individual differences in treatment and physiological status between animals and thus, a less homogenous model.

It could also be reasoned that with the magnitude of these circulatory changes, the trending ability is anticipated to be aligned. However, we believe that it is of utmost importance to challenge new monitoring technologies in extreme conditions which is ethically not possible to induce in humans.

In conclusion $\mathrm{CO}_{\mathrm{EPBF}}$ maintained a good agreement during hypercapnia and was marginally affected during severe ischemia. Only $5 \mathrm{~min}$ after massive reperfusion agreement returned to previous acceptable levels. Clinical studies on $\mathrm{CO}_{\mathrm{EPBF}}$ are underway.

Acknowledgement The faculty at the Hedenstierna laboratory in Uppsala, Sweden.

Author contributions TS, TÖ, CHS, FSS, MH, AO and HB: Study design, data collection and analysis. TS, CHS, HB: Drafting and revision of the manuscript. ER: Data collection and revision of manuscript. MW: Study design and revision of manuscript. AO, MH, FSS: Critical revision of the manuscript.

Funding This project is a collaboration between Karolinska Institutet and Maquet Critical Care AB. The work was supported by grants from Maquet Critical Care $\mathrm{AB}$, the regional agreement on medical training and research (ALF) between Stockholm County Council and the Karolinska Institute and the HMT project (Health, Medicine and Technology), a collaboration project between the Stockholm County Council and the Royal Institute of Technology (Grant Nos: 20140430, 20150910).

\section{Compliance with ethical standards}

Conflict of interest Magnus Hallbäck and Mats Wallin are employed at Maquet Critical Care AB (MCC). Fernando Suarez Sipmann performs consultant activities for MCC. Håkan Björne and Caroline Hällsjö Sander has received grants for research from MCC. Thorir Sigmundsson, Tomas Öhman, Eider Redondo, Anders Oldner declare no conflict of interest.

Ethical approval The study was approved by the Uppsala animal research ethical committee (nr. C 47/15) and performed at the Hedenstierna laboratory in Uppsala University, Sweden. All applicable international, national, and/or institutional guidelines for the care and use of animals were followed.

Open Access This article is distributed under the terms of the Creative Commons Attribution 4.0 International License (http:// creativecommons.org/licenses/by/4.0/), which permits unrestricted use, distribution, and reproduction in any medium, provided you give appropriate credit to the original author(s) and the source, provide a link to the Creative Commons license, and indicate if changes were made. 


\section{References}

1. Marik PE. Perioperative hemodynamic optimization: a revised approach. J Clin Anesth. 2014;26(6):500-5. doi:10.1016/j. jclinane.2014.06.008.

2. Waldron NH, Miller TE, Gan TJ. Perioperative goal-directed therapy. J Cardiothorac Vasc Anesth. 2014;28(6):1635-41. doi:10.1053/j.jvca.2014.07.008.

3. Gurgel ST, do Nascimento Jr P. Maintaining tissue perfusion in high-risk surgical patients: a systematic review of randomized clinical trials. Anesth Analg. 2011;112(6):1384-91. doi:10.1213/ANE.0b013e3182055384.

4. Ebm C, Cecconi M, Sutton L, Rhodes A. A cost-effectiveness analysis of postoperative goal-directed therapy for highrisk surgical patients. Crit Care Med. 2014;42(5):1194-203. doi:10.1097/CCM.0000000000000164.

5. Giglio MT, Marucci M, Testini M, Brienza N. Goal-directed haemodynamic therapy and gastrointestinal complications in major surgery: a meta-analysis of randomized controlled trials. Br J Anaesth. 2009;103(5):637-46. doi:10.1093/bja/aep279.

6. Huisman MG, Kok M, de Bock GH, van Leeuwen BL. Delivering tailored surgery to older cancer patients: preoperative geriatric assessment domains and screening tools-a systematic review of systematic reviews. Eur J Surg Oncol. 2016. doi:10.1016/j.ejso.2016.06.003.

7. Moonesinghe SR, Mythen MG, Grocott MP. High-risk surgery: epidemiology and outcomes. Anesth Analg. 2011;112(4):891901. doi:10.1213/ANE.0b013e3181e1655b.

8. Thiele RH, Bartels K, Gan TJ. Cardiac output monitoring: a contemporary assessment and review. Crit Care Med. 2015;43(1):177-85. doi:10.1097/CCM.0000000000000608.

9. Thiele RH, Bartels K, Gan TJ. Inter-device differences in monitoring for goal-directed fluid therapy. Can J Anaesth. 2015;62(2):169-81. doi:10.1007/s12630-014-0265-z.

10. Gedeon A, Forslund L, Hedenstierna G, Romano E. A new method for noninvasive bedside determination of pulmonary blood flow. Med Biol Eng Comput. 1980;18(4):411-8.

11. Gedeon A, Krill P, Osterlund B. Pulmonary blood flow (cardiac output) and the effective lung volume determined from a short breath hold using the differential Fick method. J Clin Monit Comput. 2002;17(5):313-21. doi:10.1023/A:1021232624767.

12. Peyton PJ, Thompson D, Junor P. Non-invasive automated measurement of cardiac output during stable cardiac surgery using a fully integrated differential $\mathrm{CO}(2)$ Fick method. J Clin Monit Comput. 2008;22(4):285-92. doi:10.1007/ s10877-008-9131-2.

13. Hällsjö Sander C, Hallback M, Wallin M, Emtell P, Oldner A, Bjorne $\mathrm{H}$. Novel continuous capnodynamic method for cardiac output assessment during mechanical ventilation. $\mathrm{Br} \mathrm{J}$ Anaesth. 2014;112(5):824-31. doi:10.1093/bja/aet486.

14. Hällsjö Sander C, Hallback M, Suarez Sipmann F, Wallin M, Oldner A, Bjorne H. A novel continuous capnodynamic method for cardiac output assessment in a porcine model of lung lavage. Acta Anaesthesiol Scand. 2015. doi:10.1111/ aas.12559.

15. Sander $\mathrm{CH}$, Sigmundsson T, Hallback M, Sipmann FS, Wallin $\mathrm{M}$, Oldner A, Bjorne H. A modified breathing pattern improves the performance of a continuous capnodynamic method for estimation of effective pulmonary blood flow. J Clin Monit Comput. 2016. doi:10.1007/s10877-016-9891-z.

16. Peyton P. Minimally invasive monitoring of cardiac output and lung gas exchange: taking it mainstream. J Clin Monit Comput. 2016. doi:10.1007/s10877-016-9866-0.

17. Sigmundsson T, Öhman T, Redondo E, Hallbäck M, Wallin M, Suarez Sipmann F, Oldner A, Hällsjö Sander C, Björne H.
A capnodynamic method for monitoring effective pulmonary blood flow-evaluation during hypercapnia. Intensive Care Med Exp. 2016;4(Suppl 1):A315.

18. Berggren $\mathrm{S}$. The oxygen deficit of arterial blood caused by nonventilation parts of the lung. Acta Physiol Scand. 1942;4(Suppl 2):1-92.

19. Tusman G, Sipmann FS, Bohm SH. Rationale of dead space measurement by volumetric capnography. Anesth Analg. 2012;114(4):866-74. doi:10.1213/ANE.0b013e318247f6cc.

20. Critchley LA, Yang XX, Lee A. Assessment of trending ability of cardiac output monitors by polar plot methodology. J Cardiothorac Vasc Anesth. 2011;25(3):536-46. doi:10.1053/j. jvca.2011.01.003

21. Montenij LJ, Buhre WF, Jansen JR, Kruitwagen CL, de Waal EE. Methodology of method comparison studies evaluating the validity of cardiac output monitors: a stepwise approach and checklist. Br J Anaesth. 2016;116(6):750-8. doi:10.1093/bja/ aew094.

22. Hapfelmeier A, Cecconi M, Saugel B. Cardiac output method comparison studies: the relation of the precision of agreement and the precision of method. J Clin Monit Comput. 2015. doi:10.1007/s10877-015-9711-x.

23. Cecconi M, Rhodes A, Poloniecki J, Della Rocca G, Grounds RM. Bench-to-bedside review: the importance of the precision of the reference technique in method comparison studies-with specific reference to the measurement of cardiac output. Crit Care. 2009;13(1):201. doi:10.1186/cc7129.

24. Transonic Systems Inc. Transonic precision flowprobes: perivascular flowprobe specifications. Technical Report (2011).

25. Yang XX, Critchley LA, Rowlands DK, Fang Z, Huang L. Systematic error of cardiac output measured by bolus thermodilution with a pulmonary artery catheter compared with that measured by an aortic flow probe in a pig model. J Cardiothorac Vasc Anesth. 2013;27(6):1133-9. doi:10.1053/j.jvca.2013.05.020.

26. Kirkeby-Garstad I, Tronnes H, Stenseth R, Sellevold OF, Aadahl $\mathrm{P}$, Skogvoll E. The precision of pulmonary artery catheter bolus thermodilution cardiac output measurements varies with the clinical situation. J Cardiothorac Vasc Anesth. 2015;29(4):8818. doi:10.1053/j.jvca.2014.12.016.

27. Bland JM, Altman DG. Agreement between methods of measurement with multiple observations per individual. J Biopharm Stat. 2007;17(4):571-82. doi:10.1080/10543400701329422.

28. Bland JM, Altman DG. Statistical methods for assessing agreement between two methods of clinical measurement. Lancet. 1986;1(8476):307-10.

29. Critchley LA, Critchley JA. A meta-analysis of studies using bias and precision statistics to compare cardiac output measurement techniques. J Clin Monit Comput. 1999;15(2):85-91.

30. Peyton PJ, Chong SW. Minimally invasive measurement of cardiac output during surgery and critical care: a meta-analysis of accuracy and precision. Anesthesiology. 2010;113(5):1220-35. doi:10.1097/ALN.0b013e3181ee3130.

31. Axiak Flammer SM, Critchley LA, Weber A, Pirbodaghi T, Brinks H, Vandenberghe S. Reliability of lithium dilution cardiac output in anaesthetized sheep. Br J Anaesth. 2013;111(5):833-9. doi:10.1093/bja/aet220.

32. Critchley LA, Lee A, Ho AM. A critical review of the ability of continuous cardiac output monitors to measure trends in cardiac output. Anesth Analg. 2010;111(5):1180-92. doi:10.1213/ ANE.0b013e3181f08a5b.

33. Löwy A, v Schrötter H. Untersuchungen uber die Blutcirkulation beim Menschen. Zeitschr $\mathrm{f}$ exp Pathol u Therapie. 1905;1:197-311.

34. Weinbroum AA, Biderman P, Soffer D, Klausner JM, Szold O. Reliability of cardiac output calculation by the fick principle and central venous oxygen saturation in 
emergency conditions. J Clin Monit Comput. 2008;22(5):361-6. doi:10.1007/s10877-008-9143-y.

35. Capek JM, Roy RJ. Noninvasive measurement of cardiac output using partial $\mathrm{CO} 2$ rebreathing. IEEE Trans Biomed Eng. 1988;35(9):653-61.

36. Peyton PJ. Pulmonary carbon dioxide elimination for cardiac output monitoring in peri-operative and critical care patients: history and current status. J Healthc Eng. 2013;4(2):203-22. doi:10.1260/2040-2295.4.2.203.
37. Jaffe $\mathrm{MB}$. Partial $\mathrm{CO}_{2}$ rebreathing cardiac output-operating principles of the NICO system. J Clin Monit Comput. 1999;15(6):387-401.

38. Reuter DA, Huang C, Edrich T, Shernan SK, Eltzschig HK. Cardiac output monitoring using indicator-dilution techniques: basics, limits, and perspectives. Anesth Analg. 2010;110(3):799811. doi:10.1213/ANE.0b013e3181cc885a. 